\title{
The nature of the unassociated 2FGL sources
}

\author{
T. Pursimo ${ }^{1}$, R. Ojha ${ }^{2}$, E. Ferrara ${ }^{2}$, F. Acero ${ }^{3}$, H. Johnston ${ }^{4}$ and \\ O. Titov ${ }^{5}$ \\ ${ }^{1}$ Nordic Optical Telescope, Santa Cruz de Tenerife, Spain \\ email: tpursimo@not.iac.es \\ ${ }^{2}$ UMBC/NASA Goddard Space Flight Center, USA ${ }^{3}$ Laboratoire AIM, CEA Saclay, France \\ ${ }^{4}$ Sydney Institute for Astronomy, Australia ${ }^{5}$ Geoscience Australia, Australia
}

\begin{abstract}
The majority of Fermi-LAT detected (2FGL) sources are AGN, mostly blazars. However, the second largest category in the 2FGL are unassociated sources ( $\sim 30 \%$ or 575 sources), whose multi-wavelength counterpart is either inconclusive or absent. Follow-up observations and archival data at X-ray, optical, and radio frequencies suggest that many unassociated 2FGL sources are strong candidates to be AGN. Typical observed characteristics of 2FGL detected AGN include variability at all frequencies and a spectral energy distribution (SED) with two "bumps"; a low-frequency synchrotron peak in the radio to optical/X-ray region and a highfrequency peak, possibly due to synchrotron self-Compton or Inverse Compton processes, that extends up to $\mathrm{TeV}$ energies. We present optical follow-up observations of a sample of Fermi unassociated sources with one or more potential X-ray counterparts detected within the LAT error circle.
\end{abstract}

Keywords. galaxies: active, galaxies: photometry, galaxies: absorption lines, galaxies: emission lines, gamma rays: observations, X-rays: galaxies, radio continuum: galaxies

\section{Introduction}

Understanding the nature of $\gamma$-ray sources detected by the Large Area Telescope (LAT) instrument aboard the Fermi satellite is of profound scientific interest not only because it leads to the identification of poorly studied types of known classes of objects (such as the high Compton dominance AGN) improving our chances of understanding their behaviour, but also because it holds the possibility of the discovery of new and exotic phenomena.

The sample for the observations reported here were chosen from the 555 unassociated 2FGL sources in the following manner. Our targets are bright $\gamma$-ray sources $(2 \mathrm{FGL} \sigma>$ $10)$, outside the Galactic plane $(|b|>5$ degrees), lacking previous optical data. All sources have at least one Swift X-ray detection in their 2FGL error circle. For seven sources we also have medium-deep ATCA radio follow-up in six frequencies (5.5-40 GHz) and their possibile identification was discussed by Acero et al. (2013). To these, we have added four targets so our sample consists of 11 sources.

\section{Observations and results}

Using the SDSS and GSC2.3 catalogs we got unambiguous optical identification for two targets. The other targets are too faint or in fields too crowded for firm identification. We have carried out optical followup observations using the Nordic Optical Telescope (Canary Islands), NTT (La Silla) and Gemini South. These telescopes were used for imaging to pinpoint the optical counterpart, make photometry observations, and to build 
broad band SED. Low resolution spectroscopy was also performed on some of our sample sources, to determine the optical classification of the targets.

Using archival and our optical imaging data, we managed to identify ten of the main candidates. The typical magnitudes range from $R \sim 17.4$ to 20.5 mag with a median of 19.7. Two targets were much fainter: one with $i=23.1 \mathrm{mag}$ and the other where no optical identification could be made, J0523-2530. For spectroscopic identification, we found one source from the SDSS and for four targets we used our own data. Of these, four have featureless spectra and classified as BL Lac objects, while one with narrow line Seyfert 1 (NLSy1) type spectrum (2FGL J0803.2-0339, $z=0.365$ ). Of the remaining six targets, four are bright enough for spectroscopy using medium size optical telescopes.

Using optical multi colour data combined with the Acero et al. (2013) data we build broad band SEDs for six targets. The synchrotron peak frequency distribution of these sources is similar to the 2FGL AGN distribution. Finally, we detected significant, 0.3-0.5 mag, optical variability for three out of five targets for which we had multi epoch optical data. Two more targets show variability when our recent data are combined with the archival data.

\section{2FGL vs unidentified 2FGL}

On average our sources have $5.5 \mathrm{GHz}$ fluxes between 8 and $97 \mathrm{mJy}$. In comparison, the $8.5 \mathrm{GHz}$ median flux density for 2FGL BL Lacs is $86 \mathrm{mJy}$ and $581 \mathrm{mJy}$ for FSRQs (Nolan et al. 2012). Similarly, in the optical band, our targets are from the faint end of the 2FGL distribution. Typical 2FGL BL Lacs have $R \sim 17.5$ and FSRQs $R \sim 18.0$ (Ackermann et al. 2011) about two magnitudes brighter than our average magnitude. Also, most likely we are probing slightly different parameter range than, for example, the Massaro et al. (2012) 2FGL unassociated sample since our sources are faint WISE sources, with five WISE detections.

The identification statistics is tentatively different to the identified 2FGL AGN source distribution, which has about the same ratio of BL Lac-objects and FSRQs and only a tiny fraction, 4 altogether, NLSy1s (Ackermann et al. 2011). However, the average broad band SEDs of our targets do not differ from that of the 2FGL AGNs. Also, it is interesting to note that the NLSy1 source, 2FGL J0803.2-0339, is classified as a non-blazar candidate by Paggi et al. (2013).

\section{Summary}

We have carried out optical follow-up observations of a sample of 2FGL unidentified sources. Optical spectroscopy and multicolour data suggest that most, if not all, are indeed AGN. Our sources are relatively weak, both in radio and optical, in comparison to the identified 2FGL AGN. From our small sample we can tentatively conclude that our faint 2FGL sources have SEDs similar to the brighter, identified 2FGL sources. However, the emission lines for our sample appear to be relatively weak.

\section{References}

Acero, F., et al. 2013, ApJ, 779, 133

Ackermann, M., et al. 2011, ApJ, 743, 171

Massaro F., et al. 2012, ApJ, 750, 138

Nolan, P. L., et al. 2012, ApJS, 199, 31

Paggi, A., et al. 2013, ApJS, 209, 9 